Editorial

\title{
Ethics and health in WHO Regional Office for the Eastern Mediterranean
}

\author{
Ala Alwan ${ }^{1}$
}

The constitution of WHO states that, "The enjoyment of the highest attainable standard of health is one of the fundamental rights of every human being". Ethics therefore underpin the work ofWHO. Governments too have an ethical responsibility to protect and promote the health their peoples at the public health level through the provision of appropriate and adequate health and social services, as do health care professionals working at the individual and community level in primary health centres and hospitals in both the public and private sector.

Health research involving humans is essential to the advancement of health but to safeguard the participants, ethical principles have been laid down for its conduct, such as informed consent, confidentiality and protection of privacy and ethical clearance of research by a qualified and authorized ethics committee.

The scope of ethics within health, health care delivery and health advancement is diverse and varying. Developments and advancements in medicine and medical practice as well as the emergence of new diseases and re-emergence of old ones can raise important questions that require ethical consideration. The HIV/AIDS epidemic brought up issues of equitable access to HIV services and treatment, as well as testing and counselling. Outbreaks and emergencies have raised issues such as prioritization of access to health care resources when demand is increased and shortages may occur, responsibilities of healthcare workers in light of the risks to their own health and safety, and the balance between reducing disease spread by isolation and travel restrictions and protecting the individual right to free movement. During the recent Ebola outbreak in West Africa, several other ethical issues have been raised concerning the use of unregistered interventions, the equitable distribution of such interventions and data collection while providing optimal care with a scarcity of resources. Public health surveillance has raised concerns of informed consent and the provision of standards of care, while human organ and tissue transplantation requires consideration of issues like sources and allocation of organs.

Within its ethics mandate, WHO is required to provide leadership and guidance to Member States on a wide range of public health ethics issues and support them in handling bioethics issues arising in their own countries. WHO articulates ethical and evidence-based policy options, promotes ethics-based approaches to health protection and promotion, and supports capacity-building to address ethical issues in public health nationally, regionally and globally.

A vital aspect of health research, and an ethical obligation for researchers, is the reporting of research findings in order to provide data and evidence for knowledge translation which may advance health and inform and guide policy-makers. The Eastern Mediterranean Health Journal (EMHJ) has been contributing to the dissemination of health research for 21 years and has endeavoured to maintain high ethical standards for the research articles it publishes, following established international ethical standards and guidelines on the conduct and publication of health research. However, it is apparent that those submitting papers to EMHJ are not always aware of their ethical obligations. Therefore, in order to articulate EMHJ's position on the conduct and reporting of research, and with the support and guidance of the Editorial Board, EMHJ has developed the Guidelines on the Ethical Conduct and Publication of Health Research. These aim to provide health researchers with information on the ethical standards they are expected to conform to in their papers submitted for consideration for publication.

The WHO Regional Office for Eastern Mediterranean is committed to enhancing bioethics capability in countries of the Region. In that regard and to inform readers of this work, we are pleased to publish in this issue of EMHJ three pieces related to ethics and health in the Region: a report on the work of the Eastern Mediterranean Research Ethics Review Committee; an overview of ethics in health practice and research in the Region; and the EMHJ Guidelines on the Ethical Conduct and Publication of Health Research. 